\title{
A fully automatic 3-D analysis tool for expansion chamber mufflers
}

\author{
R SRINIVASANand M L MUNJAL \\ Department of Mechanical Engineering, Indian Institute of Science, Bangalore \\ 560012, India \\ e-mail: munjal@mecheng.iisc.ernet.in
}

MS received 15 April 1997; revised 7 August 1997

\begin{abstract}
The matrix condensation technique in conjunction with the substructuring principle has been previously used to model complex commercial automotive mufflers. Though complete 3-D finite element modelling is partially eliminated, it still requires the nodal-coordinate data and connectivity data of the elements forming the segment of each substructure. It also demands the connectivity of the degrees of freedom left after matrix condensation. Thus, the data preparation phase is tedious and cannot be claimed to be simple. Moreover, the existing FEM code requires the user to have a knowledge of acoustic theory and finite element modelling. This paper describes a way to completely eliminate the data preparation phase. Given the geometric dimensions of the muffler as input, the muffler performance is obtained as the output. The results obtained are compared with analytical and experimental results for simple as well as extended-tube expansion chambers, with or without an offset.
\end{abstract}

Keywords. Mufflers; duct acoustics; automatic mesh generation; finite element methods; noise control.

\section{Introduction}

Exhaust noise is a predominant source of noise in an automobile. Various types of muffler components are used to bring down the exhaust noise levels. Commercial exhaust mufflers have large cross-sections and complicated shapes. Therefore, 1-D analysis is not adequate for proper understanding of muffler performance. Thus, there is a need for a tool to perform a complete 3-D analysis of mufflers. The automotiveindustry is vibrant and fast-changing. Therefore, analysis tools used must be highly user-interactive and should be engineered to offer readymade solutions. The need for expertise on the part of the user should be eliminated so that industries need not rely upon experts to do muffler analysis. Thus, there is a need for a tool which will give muffler performance, given the geometric dimensions of the muffler. 
While generally a one-dimensional (or, plane-wave) analysis (Munjal 1987a) is adequate, higher order modes do play an important role at higher frequencies in and around expansion chambers. Several methods have been proposed by the previous researchers for 3-D analysis of mufflers (El-Sharkawy \& Nayfeh 1978; Ih \& Lee 1985; Munjal 1987b; Abom 1990; Sahasrabuddhe et al 1991). Analytical solutions proposed by researchers (El-Sharkawy \& Nayfeh 1978; Ih \& Lee 1985; Abom 1990) could be one possible solution. However, these methods cannot yield a completely automatic code as they require eigenfunctions which are not readily known for complicated shapes. Therefore, one has to resort to methods not involving eigenfunction expansions. Various numerical techniques proposed in the literature were the collocation method, finite difference method and the popular finite element method. The collocation method (Munjal 1987) is relatively simple in-so-far as the algebra and computational effort are concerned, but is deficient in dealing with fractional area ratios and complicated shapes. Therefore, the collocation method fails to be a solution for the problem on hand. Finite difference method is not suitable for very complicated and irregular geometries (Baumeister 1980). However, the finite element method is completely general as far as the complex geometry of the muffler and different properties of the medium are concerned, and hence can handle rigid/non-rigid walled chambers, and simple or extendedtube chambers with inline/offset inlet/outlet pipes (El-Sharkawy\& Nayfeh 1978;Zienkiewicz 1979; Baumeister 1980; Ih \& Lee 1985; Munjal 1987; Abom 1990; Sahasrabuddhe et al 1991).

Finite element method was extended to acousto-structural analysis by Gladwell (1965). Later, Young \& Crocker (1975) and Craggs (1976) used FEM for prediction of muffler performance. Young \& Crocker (1976) used rectangular Hermitian elements with $C_{1}$ continuity while Craggs used the axisymmetric ring element obtained from the hexahedral element. Their FEM predictions compared well with experiments. Later, Christiansen \& Krenk (1988) used a recursive finite element technique for modelling pipes and ducts. They used an axisymmetric quadrilateral element and hence their analysis is restricted to 2-D configurations. Recently, Craggs (1989) analysed branched duct networks using FEM. A truly 3-D analysis of expansion chamber mufflers incorporating the recursive FEM technique of Christiansen \& Krenk (1988) was carried out by Sahasrabudhe et al, (1991) who used the sub-structuring principle in conjunction with the matrix condensation technique for modelling simple/extended tube expansion chamber mufflers. Though Sahasrabudhe $\boldsymbol{e t}$ al (1991) could analyse simple/extended tube expansion chambers, the FEM code was not user-interactive. The code requires the nodal coordinate data of the segments of the substructures, elemental connectivity data, and degrees of freedom left after condensation in addition to the dimensions of the muffler. Thus the data preparation phase is tedious and cumbersome. Moreover, the user should have expertise in acoustic theory, finite element modelling etc. Thus the need for a truly 3-D analysis tool is not fulfilled.

In this paper, the sub-structuring and the matrix condensation technique have been used to model muffler geometry. Given the dimensions of the muffler as the input, the 3-D finite element model is generated and muffler performance is obtained as the output. The results obtained using the automatic code are compared with the results available in the literature. 


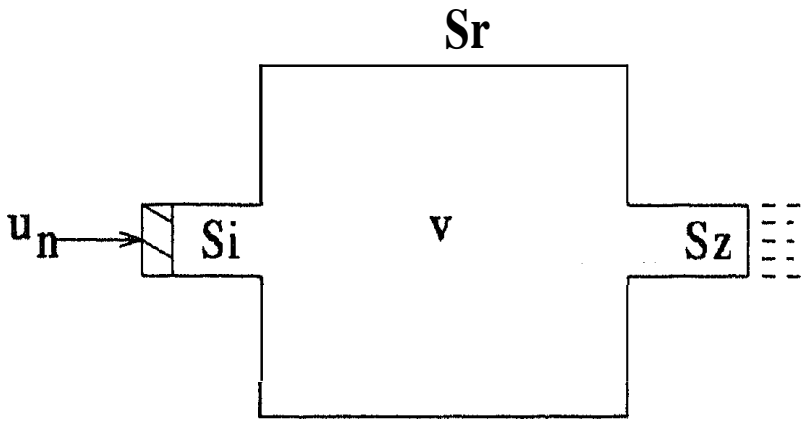

Figure 1. Acoustic system.

\section{Finite element formulation}

The acoustic field in a muffler configuration filled with stationary ideal fluid (figure 1) is given by

$$
\left[\nabla^{2}+k^{2}\right] p=0
$$

with the following four types of boundary conditions:

(1)

$$
\partial p / \partial n=-j \rho_{o} \omega(p / Z),
$$

when the normal acoustic impedance $Z$ is known;

(2)

$$
\partial p / \partial n=-j \rho_{o} \omega u_{n},
$$

when the normal particle velocity is known as in the case of the constant velocity piston source;

(3)

$$
\partial p / \partial n=0
$$

at the rigid walls of the muffler; and

$$
P=p_{n},
$$

when the constant pressure source is assumed.

Assuming a trial solution for the field variable $p$, at any point within an element as

$$
p=\{N\}_{e}^{T}\left\{p_{n}\right\}_{e},
$$

where $N_{e}$ is the basis function or the shape function or the interpolation function. Substituting (6) into (1), we get the residual as

$$
R e=\left[\nabla^{2} N_{e}^{T}+k^{2} N_{e}^{T}\right] p_{n_{e}} .
$$

This error is orthogonalized with respect to the weighting functions to get

$$
\int_{V_{e}} N_{e} R e \mathrm{~d} v=0
$$

which can be written as

$$
\int_{V_{e}} N_{e} \nabla^{2} N_{e}^{T} p_{n e} \mathrm{~d} v+\int_{V_{e}} k^{2} N_{e} N_{e}^{T} p_{n e} \mathrm{~d} v=0
$$


Then, following Munjal (1987a), we get

$$
\begin{aligned}
& {\left[[M]_{e}-k^{2}[S]_{e}+\frac{j \rho_{o} \omega}{z}[D]_{e}\right] p_{n_{e}}=-j \rho_{o} \omega F_{e},} \\
& {[M]_{e}=\int_{V_{e}} \nabla N_{e} \nabla N_{e}^{T} \mathrm{~d} V, \quad \text { (mass matrix), }} \\
& {[S]_{e}=\int_{V_{e}} N_{e} N_{e}^{T} \mathrm{~d} V, \quad \text { (stiffness matrix), }} \\
& {[D]_{e}=\int_{S_{e}} N_{e} N_{e}^{T} \mathrm{~d} S, \quad \text { (damping matrix), }}
\end{aligned}
$$

and,

$$
\{F\}_{e}=\int_{S_{e}} u_{n} N_{e} \mathrm{~d} S, \quad \text { (forcing vector). }
$$

Here, [S] denotes the stiffness matrix and the surfaces $S_{e_{z}}, S_{e_{i}}$ and $S_{e_{r}}$ denote the portion of the boundary in which normal acoustic impedance, the input source velocity and the rigid wall boundaries are known, respectively. Surface $S_{e}$ includes $S_{e_{z}}, S_{e_{i}}$ and $S_{e_{r}}$. Equation (10) can be written after global assembly as,

$$
[K]\{p\}=\{F\}
$$

where $[K]$ is called the admittance matrix given by

$$
[K]=\sum_{i=1}^{l}[K]_{e}=\sum_{i=1}^{l}\left[[M]_{e}-k^{2}[S]_{e}+\frac{j \rho_{o} \omega}{z}[D]_{e}\right],
$$

and

$$
\{F\}=\sum_{\mathrm{i}=1}^{l}\{F\}_{e}=-j \rho_{o} \omega \sum_{\mathrm{i}=\mathrm{l}}^{1}\{F\}_{e},
$$

where $l$ denotes the number of elements in the system. The contribution to forcing vector $\{F\}$ comes only from the surface $S_{i}$ at the inlet of the muffler assuming constant velocity source and contribution to the damping matrix $\{D\}$ comes from the only surface $\mathrm{Sz}$ at the outlet of the muffler (see figure 1).

\section{Muffler performance using FEM}

Higher order modes or 3-D effects are generally limited to expansion chambers and a couple of diameters length of the inlet tube and outlet tube respectively. Therefore, a muffler composed of a volume chamber with inlet and outlet tubes (figure 2) can be simulated by a linear acoustic four-pole system (Munjal 1987a). Making use of this analogy, the acoustic state variables viz., acoustic pressure and volume velocity on the inlet side can be related to those on the outlet side through the four-pole parameters also called the transfer matrix. The transfer matrix relation can be written as

$$
\left[\begin{array}{c}
p_{n} \\
v_{n}
\end{array}\right]=\left[\begin{array}{ll}
A_{11} & A_{12} \\
A_{21} & A_{22}
\end{array}\right]\left[\begin{array}{l}
p_{1} \\
v_{1}
\end{array}\right]
$$




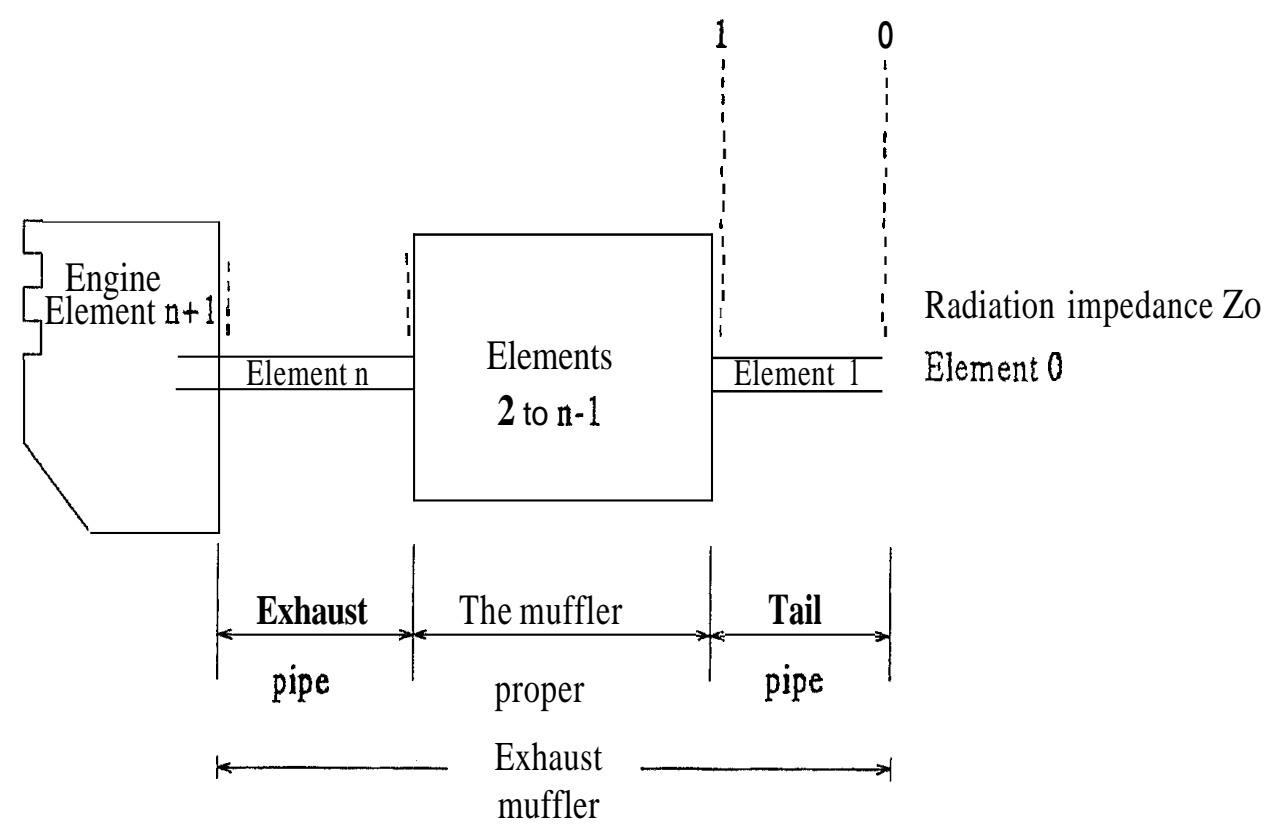

Figure 2. Engine exhaust system.

where $p$ and $v$ are, respectively, acoustic pressure and volume velocity, subscripts $n$ and 1 stand for exhaust pipe and tail pipe (figure 2) respectively, and four-pole parameters are given by

$$
\begin{aligned}
& A_{11}=\left[\frac{p_{n}}{p_{1}}\right]_{v_{1}=0}, \\
& A_{12}=\left[\begin{array}{c}
p_{n} \\
v_{1} \\
v_{1}
\end{array}\right]_{p_{1}=0} \\
& A_{21}=\left[\frac{v_{n}}{p_{1}}\right]_{v_{1}=0}, \\
& A_{22}=\left[\frac{v_{n}}{v_{1}}\right]_{p_{1}=0}
\end{aligned}
$$

The assembled set of dynamic equations, (10), obtained through finiteelement formulation, are solved twice using two sets of boundary conditions to calculate four-pole parameters.

(1) By imposing $v=0(i . e . \partial p / \partial x=0)$ at the outlet boundary, nodal points $A_{11}$ and $A_{21}$ are computed using (18) and (20).

(2) By imposing $p=0$ at the outlet boundary, nodal points $A_{12}$ and $A_{22}$ are computed using (19) and (21).

Then the transmission loss (TL) value can be evaluated as,

$$
\mathrm{TL}=20 * \log \left[\frac{1}{2} *\left|A_{11}+S_{1} \frac{A_{12}}{\rho_{o} c_{o}}+\frac{A_{21} \rho_{o} c_{o}}{S_{n}}+A_{22}\right|\right],
$$


where $S_{n}$ and $S_{1}$ are the areas of the exhaust pipe and tail pipe respectively, and $\mathrm{v}$ is the acoustic volume velocity,

$\rho_{0}=$ density of the medium,

and $c_{O}=$ sound speed.

\section{4, Selection of the finite element for muffler analysis}

In general, there is a wide-choice of finite elements available in the literature. However, the 20-noded isoparametric Brick element (Zienkiewicz 1979) has been used extensively throughout this work. The reasons for selecting Isopararnetric 20-noded brick elements are as follows.

(1) Since automotivemufflers involve curved geometry, 20-noded isoparametricelements with quadratic shape functions are found suitable. A large number of linear elements may be required to discretize the same geometry. Therefore, use of a higher-order element reduces memory requirement and solution time.

(2) 20-noded isoparametric elements have been successfully used by Sahasrabudhe et al (1991) for finite elementmodelling of mufflers (Munjal 1987a) and the results obtained are satisfactory.

\section{Maximum length of a segment and element}

Investigation of the criteria for the .maximum length of a typical segment is presented here. Ross (1981), based on his numerical experiments, reported that, in order to ensure convergence of finite element results up to a frequency of $f$-max, the maximum typical dimension of the finite element should be less than 0.2 times the minimum wavelength. We used 8-noded isopararnetric elements in his mesh configuration. Later, Sahasrabudhe et al (1991) reported that, with the use of a quadratic 3-D element (20-nodedisoparametric element), the maximum typical dimension of the finite element should be less than 0.52 times the minimum wavelength. To verify this criterion, an analytical solution was derived for a simple square pipe and it was compared with the results using FEM.

Details of the comparison are as follows:

A square pipe with its boundary conditions is shown in figure 3 . The dimensions of the pipe are such that only plane waves can propagate. Therefore, the governing equation of the problem is

$$
\left(D^{2}+k^{2}\right) p(z)=0,
$$

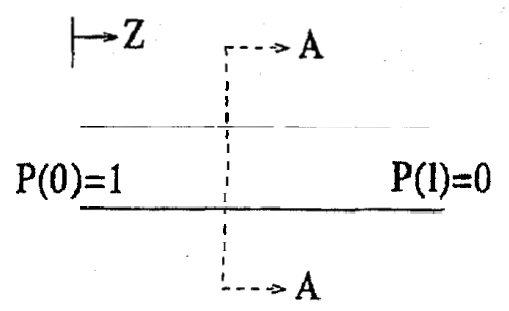

Figure 3. Square pipe model. 
and the boundary conditions are:

- @ $z=0, p(0)=1$,

- $\mathrm{z}=1, p(1)=0$.

The solution for $(23)$ is

$$
p(z)=C 1 \cdot \exp (-j k z)+C 2 \cdot \exp (+j k z)
$$

where $C 1$ and $C 2$ are constants and $k$ is the wave number. The constants $C 1$ and $C 2$ can be found by applying the two boundary conditions. Thus, the solution is given by

$$
p(z)=\sin k(l+z) \sin (k l)
$$

where

$$
l=\left(n \cdot c_{o}\right) /(4 \cdot f), \quad \text { for } \quad n=1,3,5,7 \cdots .
$$

Equation (25) is taken as a reference for validation of the FEM results.

To verify the criteria for maximum element length, the following test runs were conducted.

Data for the test run:

(1) Frequency $1000 \mathrm{~Hz}$,

(2) length $\quad l=\left(n \cdot c_{o}\right) /(4 \cdot f), n=\mathrm{I}, 3,5, \ldots$,

(3) side of the square $h<c_{o} /(2 \cdot f)$

The following procedure was adopted for conducting the test, and the steps involved are as follows:

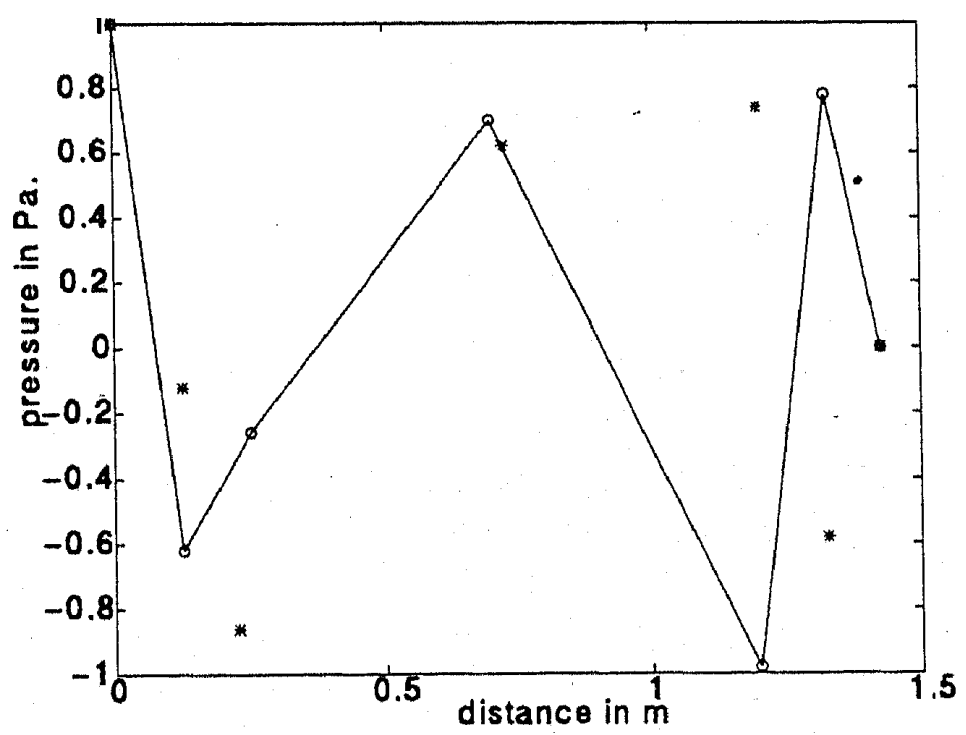

Figure 4. Comparison of FEM predictions (*) for a square pipe model of length $1.445 \mathrm{~m}$, side $0.05 \mathrm{~m}$ and a segment length 0.7 times the wavelength with analytical solution $(0)$ given by (25). 


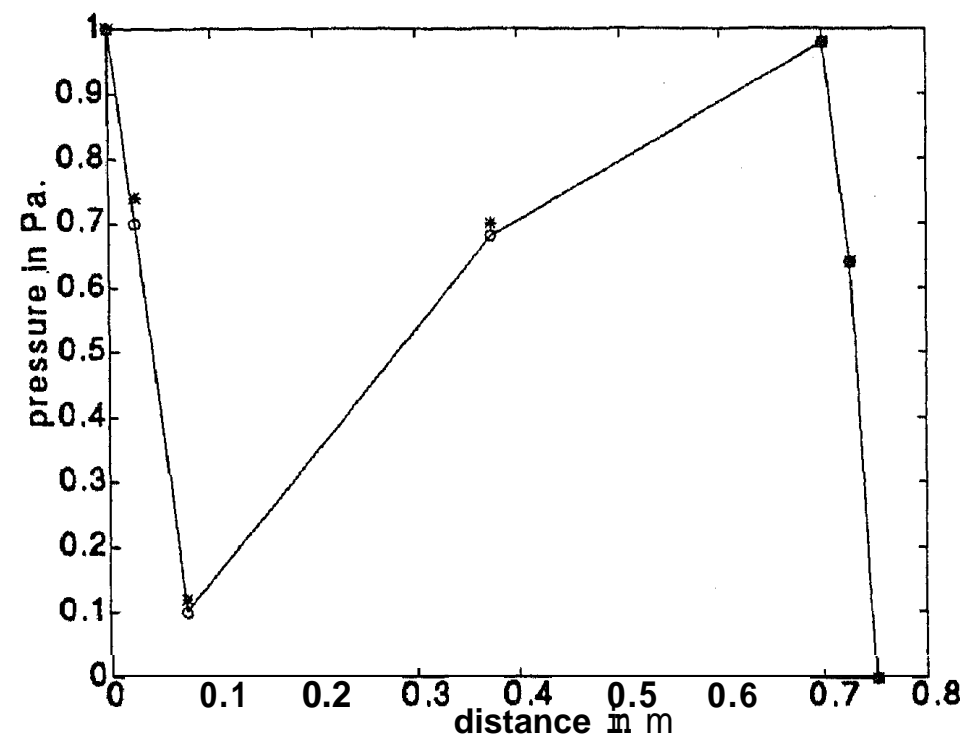

Figure 5. Comparison of FEM predictions ( $*$ ) for a square pipe model of length $0.765 \mathrm{~m}$, side $0.05 \mathrm{~m}$ and a segment length $\mathbf{0 . 2 2 5}$ times the wavelength with analytical solution (o) given by (25).

o Select a suitable value of the pipe length using (26),

$o$ vary the segment length each time (greater than 0.52 times the minimum wavelength to start with),

$\boldsymbol{o}$ the value of $h$ (side of the square cross-section) is taken equal to the segment length so that each element has an aspect ratio of unity,

$o$ compare the results with the analytical solution.

Analysis was conducted with a segment length equal to $0.7,0.54,0.375,0.225$ times the minimum wavelength. The results for two cases are shown in figures 4 and 5 . The solution obtained using 0.225 times the minimum wavelength (figure 5) tallied quite well with the analytical solution. From the above results it may be observed that the element length must be certainly less than 0.375 times the minimum wavelength.

A limitation of the foregoing analysis is that it is conducted in the plane wave zone where the effect of higher order modes is not present. Hence, to check the validity of the above tests further, a complete 3-D analysis of a simple expansion chamber muffler was performed and the computed values of TL were compared with those obtained from an analytical solution. The tests are as below.

A simple expansion chamber with a diameter ratio of 3 and length to diameter ratio of 1.67 was analysed and the transmission loss thus obtained was compared with the results available in the literature (El-Sharkawy \& Nayfeh 1978). The segment length was taken as $0.125 \mathrm{~m}$. From figure 6 , it may be observed that the results agree well up to a frequency of $865 \mathrm{~Hz}$. This observation leads to the conclusion that the segment length should be less than or equal to 0.3 times the minimum wavelength of interest. Figure 7 shows the comparison between TL of a simple expansion chamber with the same dimension as in the previous case but differing in that the segment length taken is $0.062 \mathrm{~m}$. The results agree quite well up to a non-dimensional frequency parameter $k l_{2}$ of $\mathbf{8}$ which corresponds to 


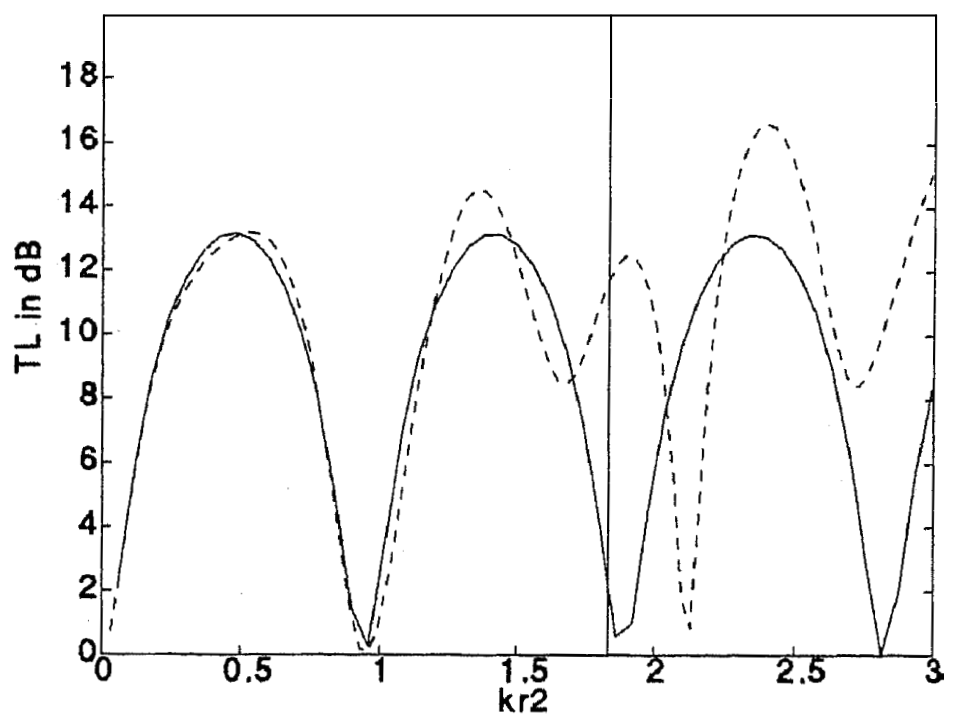

Figure 6. Comparison of TL of a coaxial expansion chamber with $l_{1}=13=0.375 \mathrm{~m}$, $d_{1}=d_{3}=0.05 \mathrm{~m}, l_{2}=0.2505 \mathrm{~m}, d_{2}=0.15 \mathrm{~m}$, and with segment length $0.125 \mathrm{~m},(--)$ FEM, $(\rightarrow$ analytical (El-Sharkawy \& Nayfeh 1978).

a frequency of $1730 \mathrm{~Hz}$. This observation confirms the previous conclusion. From these numerical experiments the following conclusions were derived.

- A 20-noded isoparametric element can be used to model pipes with curved geometry.

- Maximum typical dimension has to be less than 0.3 times the minimum wavelength when using the 20-noded isoparametric element and less than 0.2 times the minimum wavelength when using linear elements as given by Ross (1981).

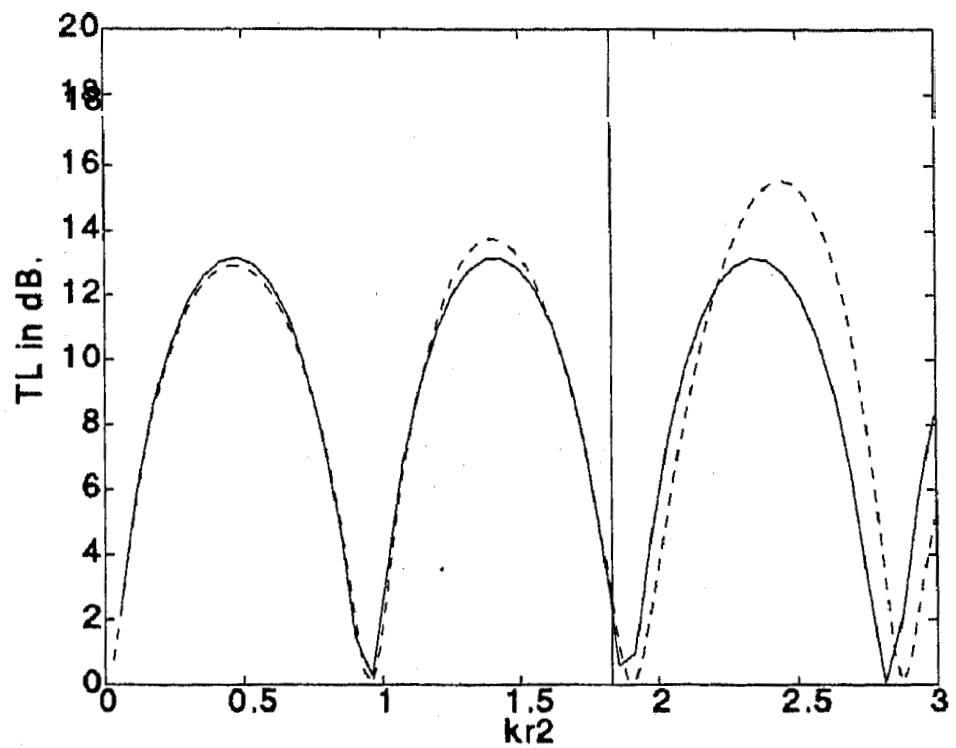

Figure 7. Comparison of TL of a coaxial expansion chamber with $l_{1}=13=0.187 \mathrm{~m}$, $d_{1}=d_{3}=0.05 \mathrm{~m}, l_{2}=0.2505 \mathrm{~m}, d_{2}=0.15 \mathrm{~m}$, and with segment length $0.062 \mathrm{~m},(--)$ FEM, $(\rightarrow$ analytical (El-Sharkawy \& Nayfeh 1978). 


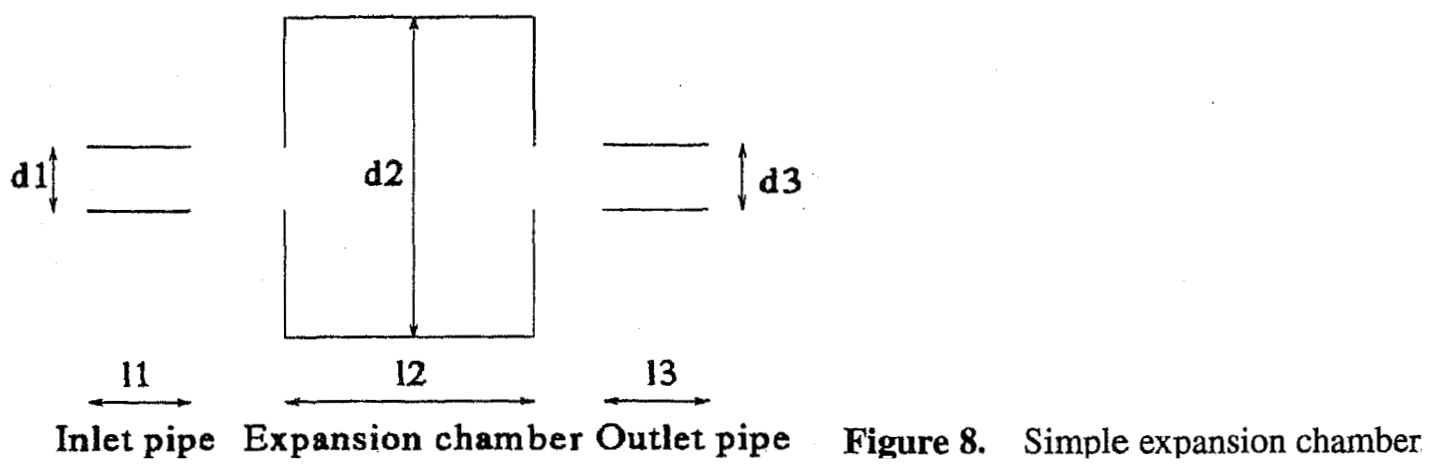

\section{Modelling of simple expansion chambers}

A typical simple expansion chamber muffler is shown in figure 8 .

Basically it consists of three substructures (Munjal 1987a):

- Inlet pipe (substructure 1);

- expansion chamber (substructure 2); and

- the outlet pipe (substructure 3).

The task of a mesh generator is to discretize the volume of the muffler. The FEM code takes the length and diameter of the inlet pipe, the expansion chamber and the outlet pipe respectively as the inputs. The basis for the finite element modelling is the matrix condensation technique (Munjal 1987a). The construction of a 3-D finite element model proceeds as follows.

Nodes are generated in a 2-D region as shown in figure 9 which uses only the inlet pipe diameter and the chamber diameter. The mesh shown is a cross-section of a typical segment of substructure2. This 2-D mesh can be extruded to form a typical segment of substructure 2 (Sahasrabudheet al 1991). The 8-nodes on the periphery of the inner circle are extruded to form pipe segments for the inlet/outlet pipes. Number of segments of substructure 2 is given by $m$ where it becomes $m+1$ for inlet/outlet pipes. The segment at the inlet section of substructure 1 and outlet section of substructure 3 are not condensed, as the gradient of pressure is computed using these segments. This is useful in the computation of four-pole parameters as discussed.
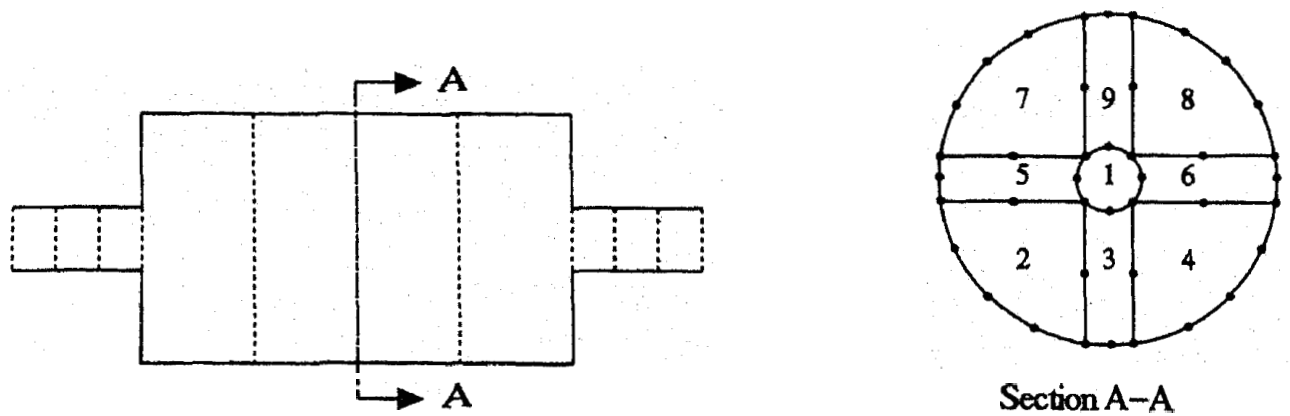

Figure 9. Simple expansion chamber discretization. 


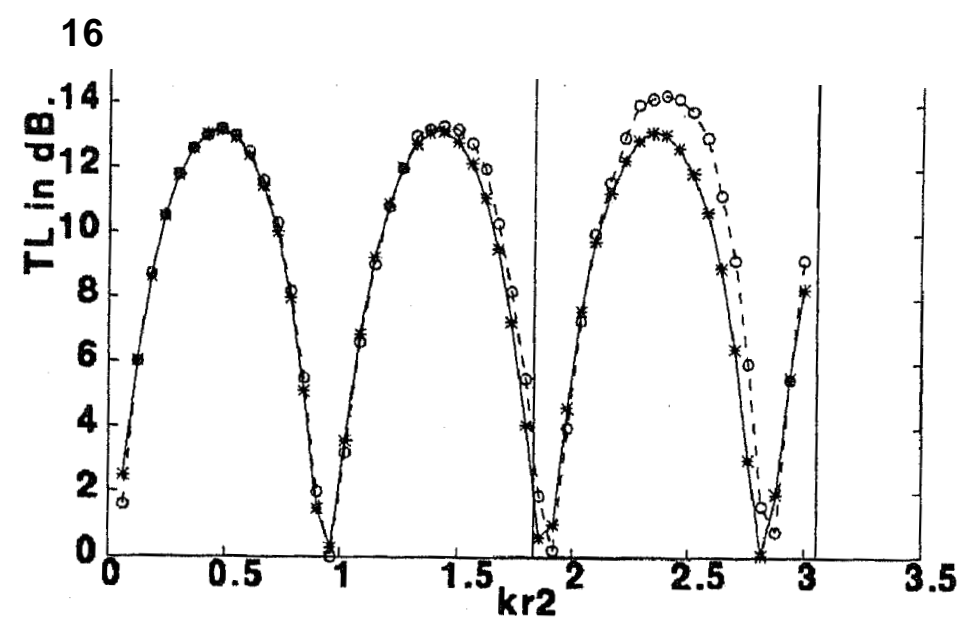

Figure 10. Comparison of FEM results for a simple expansion chamber with $d_{1}=0.05 \mathrm{~m}$, $d_{2} / d_{1}=3, l_{2} / d_{2}=1.67$ with the analytical solution of El-Sharkawy \& Nayfeh (1978). (*) analytical,(o) FEM.

Here, $m$ is any positive integer such that the ratio $1 / 2^{m}$ is less than 0.3 times the minimum wavelength. One has to note that only nine elements are generated irrespective of the muffler dimension. This mesh is found to be sufficientto analysemufflers of practical interest. Since the same number of elements are formed, the connectivity data for a typical segmentremain the same and are read from a data file. In the same manner, the information regarding the degrees of freedom left after matrix condensation is read from the data file. Thus, the geometric dimensions alone are sufficient to obtain a 3-D finite element model of a simple expansion chamber muffler. Limitations of the code are as follows.

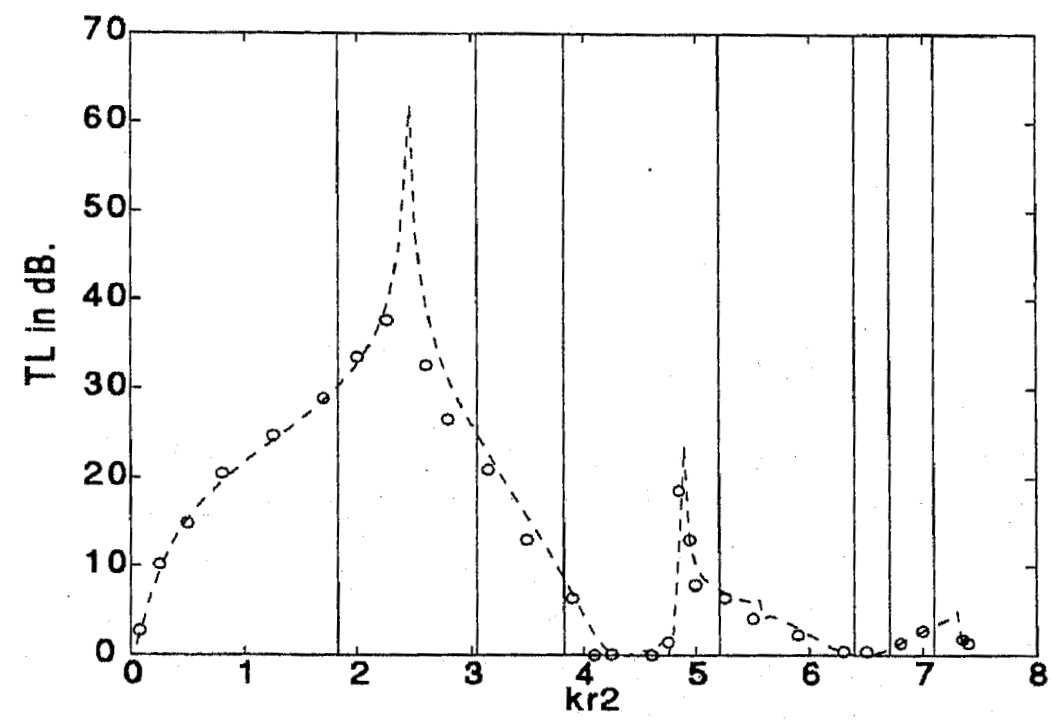

Figure 11. Comparison of the TL for a simple expansion chamber with $d_{1}=0.0205 \mathbf{m}$, $d_{2} / d_{1}=6, l_{2} / d_{2}=0.33$, with the experimental results of Th \& Lee (1985) (-- -) FEM, (o) experiments. 


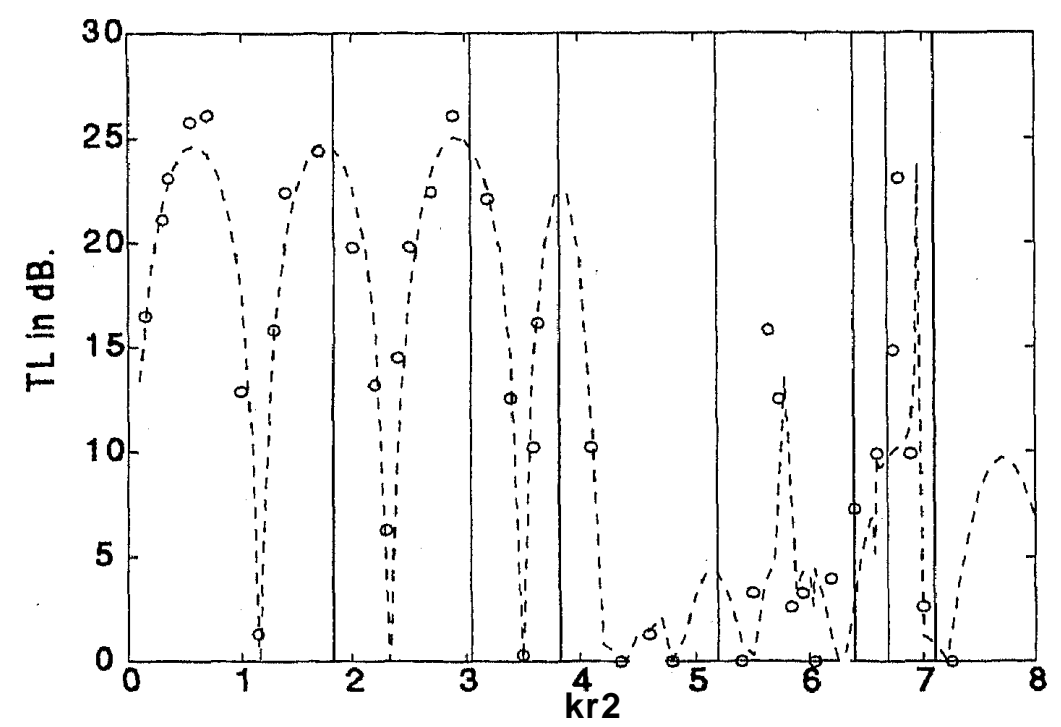

Figure 12. Comparison of the FEM predictions for coaxial expansion chamber with $d_{1}=$ $0.0205 \mathrm{~m}, d_{2} / d_{1}=6, l_{2} / d_{2}=1.35$ with the experimental results of Ih \& Lee (1985) (- -) FEM, (o) experiments.

- The circumference of the inlet/outlet pipe should be less than 1.2 times the minimum wavelength of interest.

- The differencebetween the radius of inlet pipe and expansion chamber should not exceed 0.3 times the minimum wavelength.

These limitations may easily be overcome by using an adaptive mesh generator which will create more elements for a bigger dimension so that the maximum typical dimension of any of the elements does not exceed the prescribed limit. Thus the process of finite element modelling is fully automated without user intervention.

To validate the modelling procedure discussed in the previous section, the results obtained using the completely automatic FEM code were compared with the experimental and analyticalresults availablein the literature. They are as shownin the figures 10-12. The finite element results showed good agreement with the analytical solution (El-Sharkawy \& Nayfeh 1978) throughout the frequency range of interest as can be seen in figure 10. From figures 11 and 12, it is seen that the FEM predictions are in good agreement with the experimental results as well.

\section{Modelling of extended tube expansion chambers}

A typical extended tube expansion chamber with five substructures is shown in figure 13. One may note that modelling of extended tube expansion chambers is very similar to that of simple expansion chambers. The difference lies in the additional task of modelling the two additional substructures (annular cavity introduced by the extended pipes). Figure 13 shows the discretized cross-section of the annular cavity between the inlet pipe and the chamber. The nodes are formed in much the same way as the simple expansion chamber but the differencelies in the connectivity. In the annular cavity model, the element representing the cross-section of the inlet pipe is absent. It is to be noted here that there is no interaction 


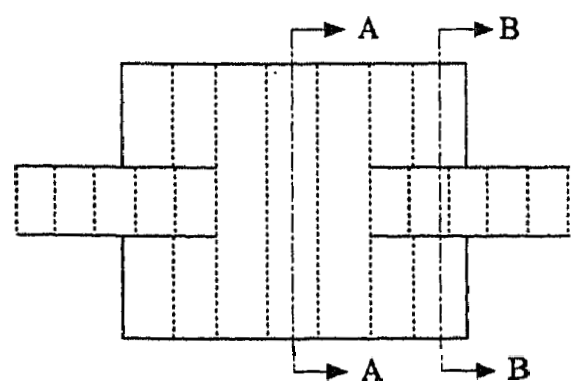

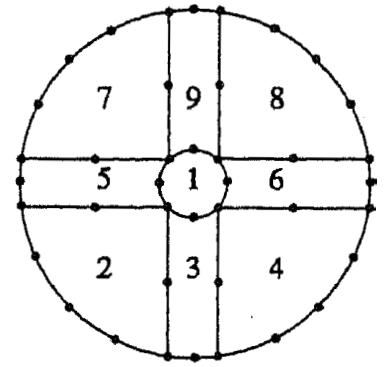

Section A-A

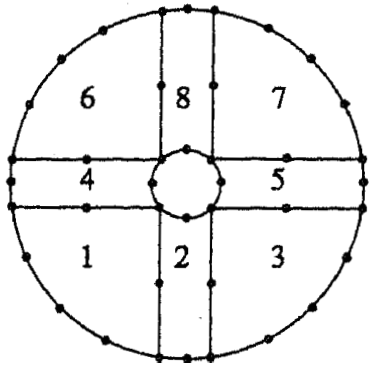

Section B-B

Figure 13. Discretized cross-sections of the annular cavity and the expansion chamber.

between the substructures 1 and 2, or 4 and 5 as the walls of the pipe are assumed to be rigid. The interaction is only through substructure 3. The input to the FEM code will be the length and diameter of the five substructures. The FEM program can handle the following cases:

- extended inlet and extended outlet,

- simple inlet and extended outlet,

- extended inlet and simple outlet.

To validate the modelling procedure illustrated above, an extended tube expansion chamber was analysed and the results were compared with the experimental results of th \& Lee (1985). The comparison is shown in figure 14.

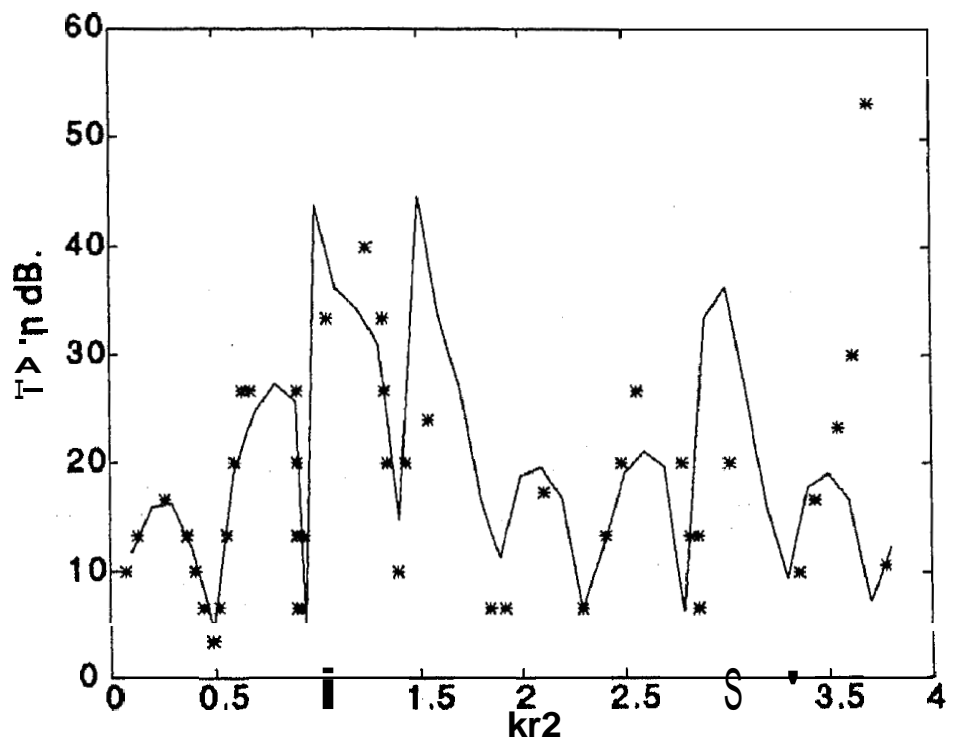

Figure 14. Comparison of the FEM predictions for an extended tube expansion chamber having $r_{1}=r_{3}=25 \mathrm{~mm} ; \alpha=3.49, \beta=3.367, l_{e i}=3.72 r_{1}, l_{e o}=5.58 r_{1}, l_{2}^{\prime}=14.2 r_{1}$ with analytical method of Abom (1990); $(*)$ analytical, $(\rightarrow$ FEM. 

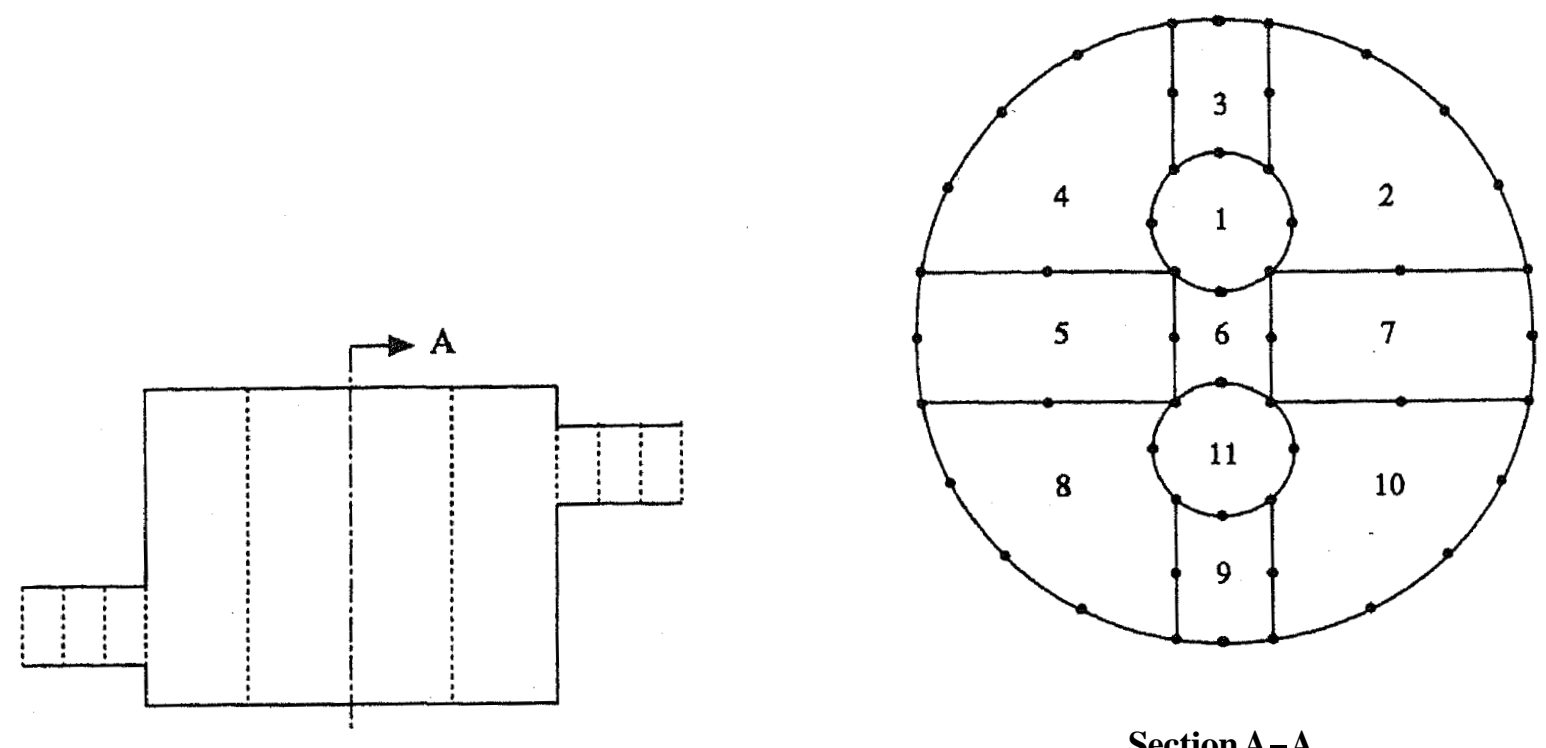

Section A-A

Figure 15. Discretized cross-section of an offset inlet/outlet model.

\section{Modelling of offset inlet/outlet chambers}

In the case of offset pipe chambers, the automatic FEM code requires as the input the centre and radii of the pipes forming the substructures. The mesh generation routines are capable of handling any arbitrary position of the inlet and outlet pipes provided the two pipes do not intersect. Figure 15 shows the mesh generated for an angular position of $180^{\circ}$ between the inlet and outlet pipes.

Two-dimensional nodal data are converted into a segment model as shown in figure 15 , which after condensation is representative of the whole volume of the muffler. Thus

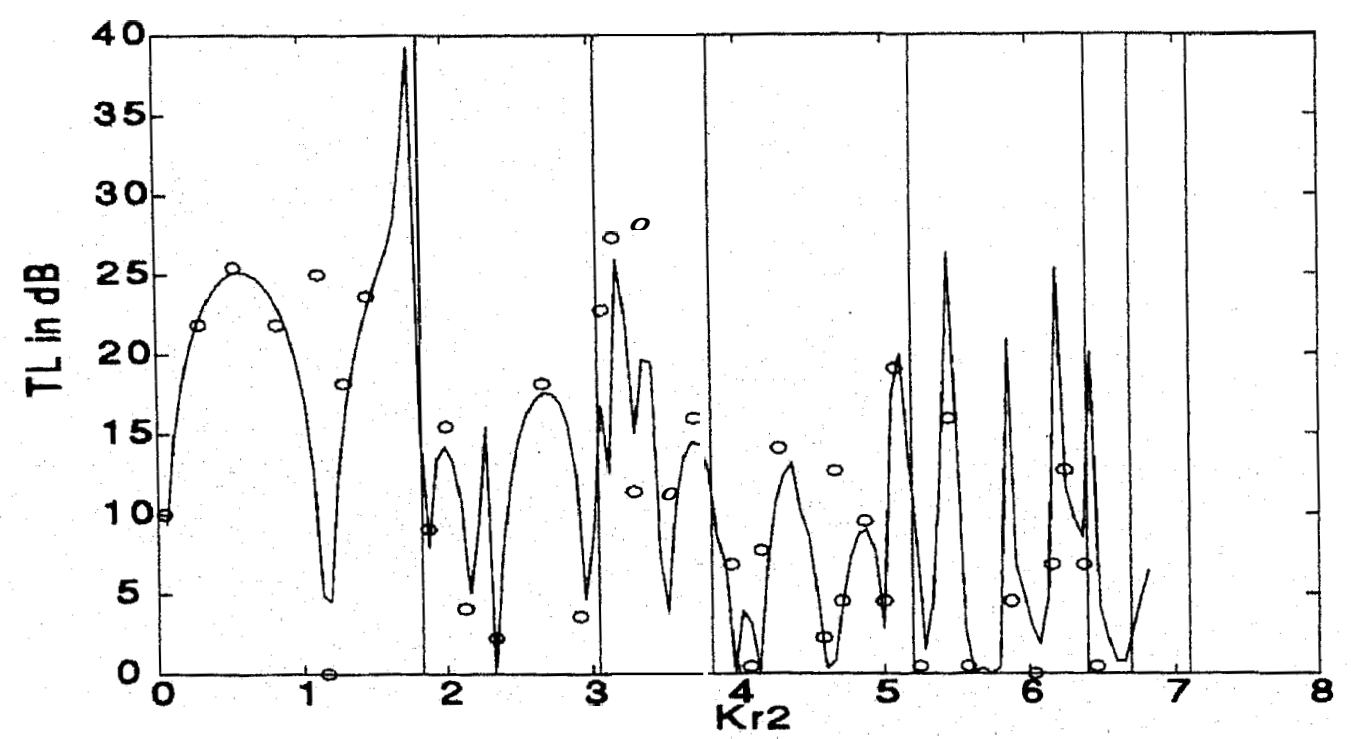

Figure 16. Comparison of the FEM predictions $(\rightarrow$ for an offsetexpansion chamber with the experimental results of Ih \& Lee (1985). $\alpha=6, \beta=1.35 ; r_{1}=r_{3}=10.25 \mathrm{~mm}$; $\delta_{1}=\delta_{2}=0.37 r_{2} ; \theta=180^{\circ}$. 


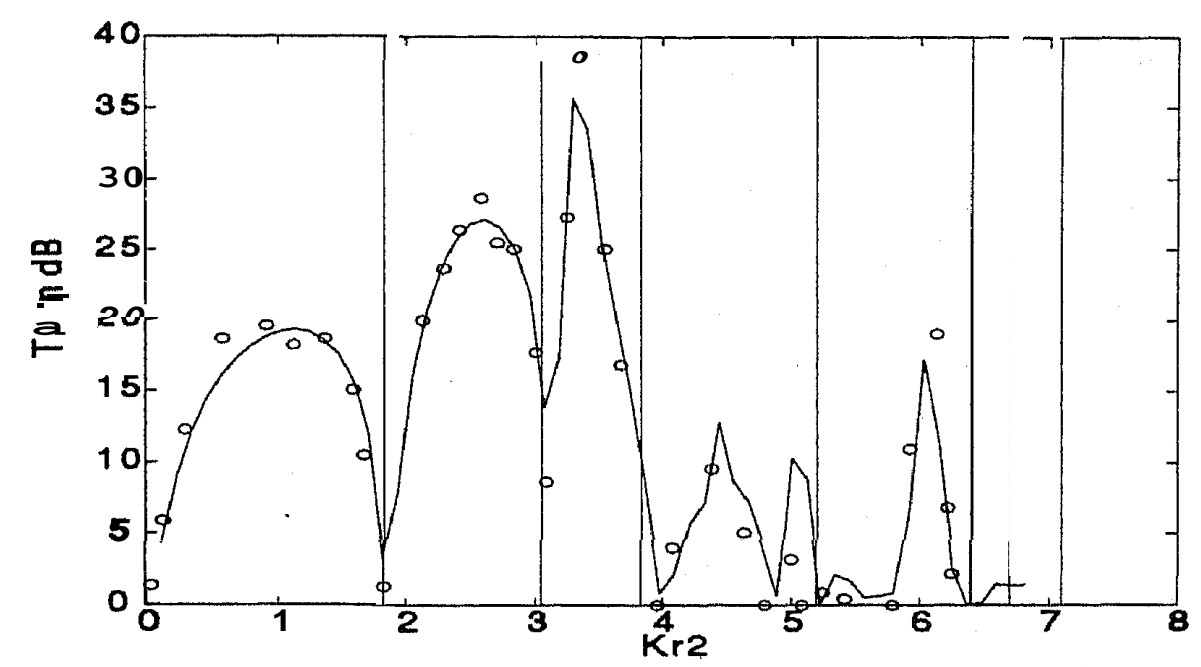

Figure 17. Comparison of FEM predictions $(\rightarrow$ for an offset expansion chamber with the experimental results of Ih \& Lee (1985). $a !=6, \beta=\mathbf{0 . 3 3} ; r_{1}=r_{3}=10.25 \mathrm{~mm}$; $\delta_{1}=\delta_{2}=0.37 r_{2} ; \theta=180^{\circ}$.

matrix condensation eliminates the task of constructing a complete 3-D model, which is quite cumbersome and consumes a lot of memory and CPU time. The nodal connectivity data for the segments of different substructures are read from a data file. The number of segments in each of the substructures is calculated in such a way that the ratio $l / 2^{m}$ (segment length) is less than 0.3 times the minimum wavelength. Here m denotes the number of successivematrix condensations(Christiansen \& Krenk 1988). Thus the process of modelling becomes fully automatic.

Figures 16 and 17 corroborate the finite element results obtained in the form of TL of acoustically long as well as short offset inlet/outlet expansion chamber mufflers with the experimental results of Ih \& Lee (1985).

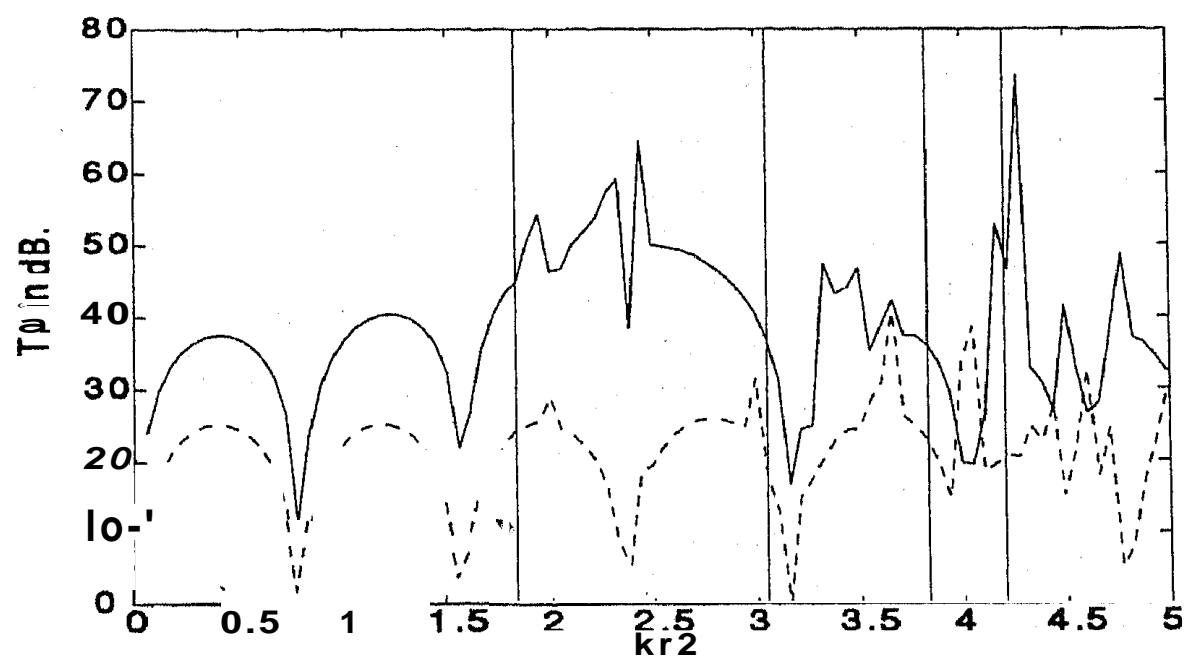

Figure 18. Comparison of the TL of the offset simple $\left.(-)^{-}\right)$and offset extended tube $(\rightarrow$ expansion chambers with $l_{e i}$ and $l_{e o}=0.33 d_{2}$ for the extended tube. $a^{\prime}=6, \beta=2 ; \delta_{1}=0$, $\delta_{2}=0.63 r_{2}, \theta=0^{\circ}$. 


\section{Modelling of offset extended inlet/outlet pipes}

The modelling of mufflers with inlet/outlet pipes offset and extended into the expansion chamber is done using the concepts discussed in the previous sections. Two more substructures are introduced by extending the inlet/outlet pipes. These cavities are modelled in the same way as the offset expansion chamber, the difference being the absence of the element corresponding to the pipe segment. While the element eleven corresponding to the inlet pipe is absent in the segment model of substructure two, element one is missing in the segment model of the substructure four (see figure 15).A typical muffler with axial inlet and radially offset outlet pipe was analysed with and without the extension of the inlet/outlet pipes and the results compared. The comparison is shown in figure 18. The resonances in the end chambers are found to improve the performance of the extended tube chambers as compared to their simple chamber counterpart.

\section{Integration of 3-D analysis with 1-D analysis}

Often, higher order modes that are generated in expansion chambers decay quickly in the adjoining inlet/outlet pipes, where only plane waves can propagate. Thus, the completely automatic 3-D analysis subroutines developed for an expansion chamber with small (about two diameters long) portions of the inlet pipe and outlet pipe can be used to generate a $2 \times 2$ transfer matrix as described above. This can then be integrated with Munjalś Transfer Matrix-based Muffler Program (TMMP) which, in fact, was the primary purpose of the investigation. Whenever expansion chambers appear as a part of the acoustic system, the 3-D analysis routines are called upon to get the four-pole parameters connecting the inlet and outlet sections of the chamber. Thereafter, respective routines of the program are used to perform plane-wave analysis. The overall performance of the acoustic system can be obtained by simply multiplying the successive transfer matrices (Munjal 1987a). As an illustration, consider an acoustic system which consists of a simple expansion chamber and a concentric tube resonator in series as shown in figure 19. The overall performance of the system is calculated and plotted in figure 20. It may be noted that 1-D analysis results show good agreement up to the cut-on of the first azimuthal mode, but show discrepanciesin the high frequency range. This observation clearly illustrates the inadequacy of plane-wave analysis above the cut-on frequencies of higher order modes. But the CPU time for each frequency stepin the case of a 3-D FEM program is at least 100times that of a plane-wave analysis program. Therefore, one has to resort to 3-D analysis only when the higher order mode effects predominate within the frequency range of interest. Thus it is possible to analyse any combination of simple/extended tube chambers in series using the TMMP

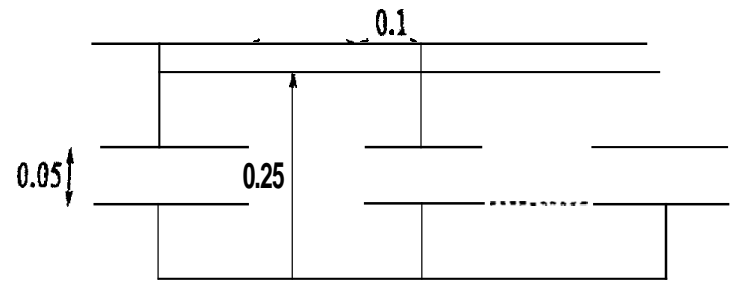

Figure 19. Extended tube expansion chamber 


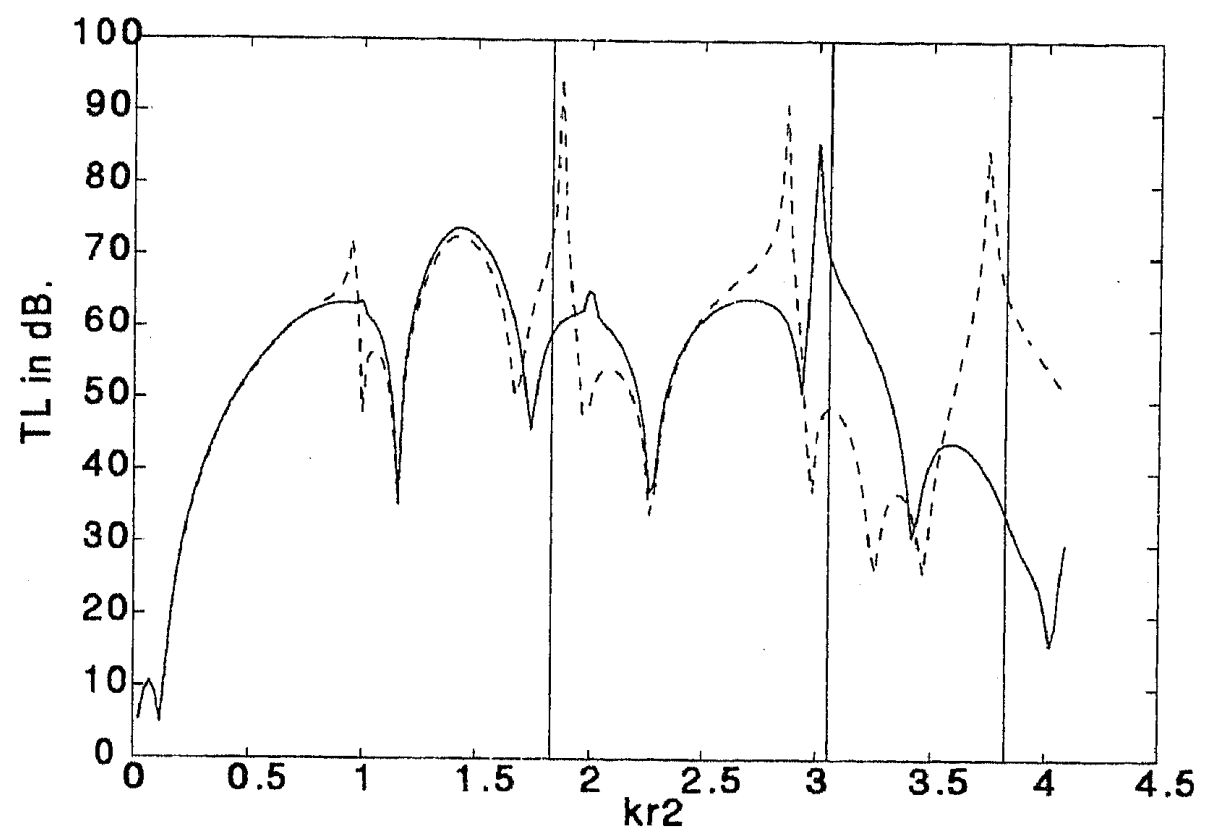

Figure 18. Comparison of FEM predictions (-- -) with TMMP(-) for the acoustic system shown in figure 19.

with 3-D analysis routines being restricted to chambers whenever the user feels that 3-D analysis is inevitable.

\section{Concluding remarks}

- Maximum typical dimension of a finite element should be less than 0.3 times the minimum wavelength of interest while using quadratic shape functions and 0.2 times the minimum wavelength for linear shape functions, as suggested by Ross (1981).

- A completely automatic FEM program which takes dimensions of the muffler as the input and four pole parameters as the output has been developed for analysing simple/extended tube expansion chambers with coaxial or offset location of the inlet/outlet pipes and integrated with Munjal's TMMP software. This work thus fulfills the need for a userfriendly tool for 3-D analysis. It is to be noted that the need for an expert with knowledge of acoustic theory and finite element modelling is totally eliminated.

\section{References}

Abom M 1990Derivation of four-pole parameters including higher order mode effects for expansion chamber mufflers with extended inlet and outlet. J. Sound. Vib. 137: 403-418

Baumeister K J 1980 Numerical techniques in linear duct acoustics. NAA-TM-81553, E-513

Christiansen P S, Krenk $S 1988$ A recursive finite element technique for acoustic fields in pipes with absorption. J. Sound. Vib. 122: 107-118

Craggs A 1976A finite element method for damped acoustic systems: an application to evaluate the performance of reactive mufflers. J. Sound. Vib. 48: 377-392 
Craggs A 1989The application of the transfer matrix and matrix condensation methods with finite elements to duct acoustics. J. Sound. Vib. 132: 393-402

El-Sharkawy I, Nayfeh A H 1978 Effect of an expansion chamber on the propogation of sound in circular ducts. J. Acoust. Soc. Am. 63: 667-674

Gladwell G M L 1965Proceedings of Fifth International Congress on Acoustics, Liege, L-33.

Gopinathan V 19893-D analysis of simple axisymmetric expansion chamber mufflers for stationary medium by the exact method. M E dissertation, Indian Inst. Sci., Bangalore

Ih J G, Lee B H 1985 Analysis of higher-order mode effects in the circular expansion chamber with mean flow. J.Acoust. Soc. Am. 77: 1377-1388

Munjal M L 1987a Acoustics of ducts and muffers (New York: John Wiley and Sons)

Munjal ML $1987 \mathrm{~b}$ A simplenumerical method for three-dimensional analysis of simpleexpansion chamber mufflers of rectangular as well as circular cross-section with stationary medium. $J$. Sound fib. 116: 71-88

Ross D F 1981 A finite element analysis of parallel-coupled acoustic systems. J. Sound. fib. 79: 133-143

Sahasrabudhe A D, Munjal M L, Anantharamu S 1991 Matrix condensation and transfer matrix techniques in the 3-D analysis of expansion chamber mufflers. J. Sound Vib. 147: 371-394

Young C I J, Crocker M J 1975 Prediction of transmission loss in mufflers by the finite element method. J. Acoust. Soc. Am. 57: 144-148

Zienkiewicz O C 1979 The finite element method 3rd edn (New Delhi: Tata McGraw-Hill) 